\title{
Association of cancer cell type and extracellular vesicles with coagulopathy in patients with lung cancer and stroke
}

Jong-Won Chung, MD, MSc ${ }^{1,4}$; Yeon Hee Cho, MS ${ }^{2,4}$; Myung-Ju Ahn, MD, PhD ${ }^{3}$; Mi Ji Lee, MD, MSc'; Gyeong-Moon Kim, MD, PhD ${ }^{1}$; Chin-Sang Chung, MD, PhD ${ }^{1}$; Oh Young Bang, $M D, P h D^{1,4}$

Departments of ${ }^{1}$ Neurology and ${ }^{3}$ Hemato-oncology, Samsung Medical Center, Sungkyunkwan University School of Medicine; ${ }^{2}$ Clinical Research Center, Samsung Biomedical Research Institute; ${ }^{4}$ Translational and Stem Cell Research Laboratory on Stroke, Samsung Medical Center

\section{BACKGROUND}

- : Coagulopathy is an important cause of stroke in cancer patients. However, underlying mechanisms and clinical factors related to coagulopathy remain unclear. Cancer cells secrete extracellular vesicles (EVs), and circulating EVs in cancer patients are reportedly associated with clinical outcomes.

- We hypothesized that certain characteristics of cancer affect coagulopathy in patients with lung cancer and ischemic stroke.

\section{METHODS}

- Consecutive patients with active lung cancer and acute ischemic stroke were prospectively studied.

- Volume and pattern of acute brain infarcts and plasma levels of circulating tumor EVs (CD326, CD133, and CD144) were measured using flow cytometry.

- In vitro experiments investigated the pathophysiological mechanisms underlying cancer-associated coagulopathy.

\section{STUDY DESI GN}

1. Clinical Study: EVs levels in cancer stroke patients
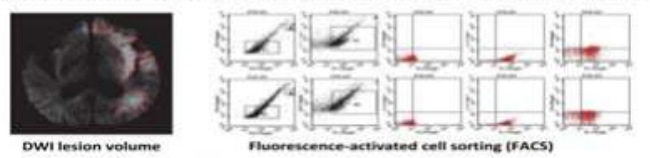

DWi lesion volume


2-1. Mechanism study: EVs from cell lines and patients
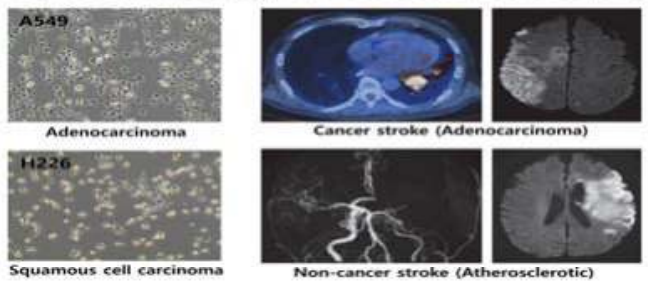

2-2. Mechanism study: Coagulation assay with EVs



\section{RESULTS}

- Of 114 patients, $95(83.3 \%)$ had an adenocarcinoma cell type and 95 $(83.3 \%)$ had distant metastasis.

- Acute brain infarct volumes were larger $(\mathrm{P}=0.009)$ and circulating EV levels were higher $(\mathrm{P}<0.01$ for all EVs $)$ in patients with an adenocarcinoma cell type than in those with other cell types.

- The presence of metastasis was not associated with infarct volume or circulating EV levels.

- Coagulation assays demonstrated dose-dependent shorter clotting times after treatment with EVs from adenocarcinoma cell lines than with use of EVs from squamous cell carcinoma.

- These findings were confirmed by coagulation assays using circulating EVs from patients with adenocarcinoma and stroke and from those with conventional stroke mechanisms.

\section{EXTRACELLULAR VESI CLE LEVELS BY CANCER TYPE} AND METASTASIS STATUS

\begin{tabular}{lllllll}
$\begin{array}{l}\text { Others } \\
(\mathrm{n}=19)\end{array}$ & $\begin{array}{l}\text { Adenocarcinoma } \\
(\mathrm{n}=95)\end{array}$ & $P$ value $^{*} \begin{array}{l}\text { Without } \\
\text { metastasis } \\
(\mathrm{n}=19)\end{array}$ & $\begin{array}{l}\text { With } \\
\text { metastasis } \\
(\mathrm{n}=95)\end{array}$ & $P$ value $\dagger$ \\
\hline $\mathrm{CD} 326$ & $0.068 \pm 0.091$ & $0.293 \pm 0.645$ & 0.002 & $0.269 \pm 0.517$ & $0.252 \pm 0.612$ & 0.900 \\
$\mathrm{CD} 133$ & $0.240 \pm 0.214$ & $0.797 \pm 1.593$ & 0.001 & $0.596 \pm 0.867$ & $0.726 \pm 1.566$ & 0.613 \\
$\mathrm{CD} 144$ & $0.011 \pm 0.016$ & $0.038 \pm 0.086$ & 0.007 & $0.027 \pm 0.041$ & $0.034 \pm 0.085$ & 0.563 \\
\hline
\end{tabular}

Values are mean \pm standard deviation.

$P$ values are calculated for adenocarcinoma vs. others* and without vs. with metastasis $\dagger$

MULTI VARI ABLE ANALYSIS: PREDI CTORS OF PLASMA EXTRACELLULAR VESI CLE LEVEL

\begin{tabular}{|c|c|c|c|c|c|c|}
\hline & \multicolumn{2}{|l|}{ CD326 } & \multicolumn{2}{|l|}{ CD133 } & \multicolumn{2}{|l|}{ CD144 } \\
\hline & $\begin{array}{l}\text { Coeffici } \\
\text { ent }\end{array}$ & $\begin{array}{l}P \\
\text { value }\end{array}$ & $\begin{array}{l}\text { Coeffici } \\
\text { ent }\end{array}$ & $\begin{array}{l}P \\
\text { value }\end{array}$ & $\begin{array}{l}\text { Coeffici } \\
\text { ent }\end{array}$ & $\begin{array}{l}P \\
\text { value }\end{array}$ \\
\hline Age (by 1-y increase) & & & 0.02 & 0.101 & & \\
\hline Hypertension & 0.07 & 0.455 & 0.19 & 0.372 & 0.01 & 0.410 \\
\hline Diabetes & & & & & 0.05 & 0.009 \\
\hline Previous stroke or TIA & 0.14 & 0.219 & & & & \\
\hline Cancer type & & & & & & \\
\hline $\begin{array}{l}\text { Adenocarcinoma } \\
\text { Others (reference) }\end{array}$ & 0.14 & 0.213 & 0.50 & 0.039 & 0.03 & 0.005 \\
\hline Distant metastasis & -0.14 & 0.216 & 0.10 & 0.972 & 0.01 & 0.496 \\
\hline $\begin{array}{l}\text { Cancer diagnosis to blood } \\
\text { sampling, } \\
\text { (by 1-d increase) }\end{array}$ & 0.01 & 0.074 & 0.001 & 0.194 & & \\
\hline Chemotherapy & & & -0.48 & 0.034 & & \\
\hline
\end{tabular}

COAGULATION ASSAY USING EVS FROM CANCER CELL LINES AND EVS FROM PATIENTS
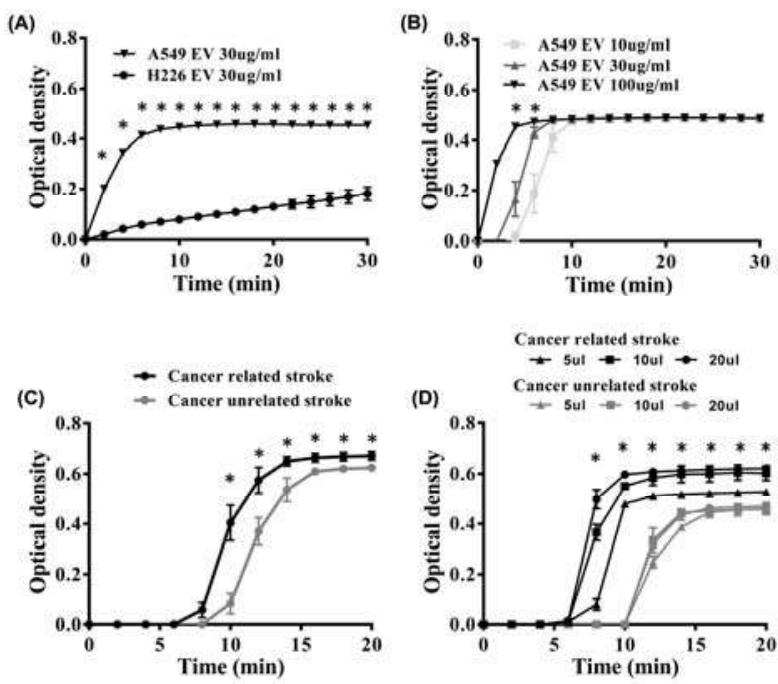

\section{CONCLUSI ON}

- Our findings indicate that cancer cell-derived EVs play an important role in coagulopathy in patients with lung cancer and stroke.

- In addition, our results showed that several clinical (i.e., cancer cell type) and therapeutic (chemotherapy) factors are associated with circulating EV levels. Further studies are needed on cargo proteins and microRNAs of EVs.

Stroke. 2018 May;49(5):1282-1285. SMMSUNG SAMSUNG MEDICAL CENTER 